\title{
Adsorção simultânea e competitividade de cádmio e cobre em solos naturais e antropizados da cidade de Cajapió, Baixada Maranhense
}

A contaminação de solos por metais potencialmente tóxicos tem provocado danos ao meio ambiente e à saúde humana nas últimas décadas. 0 acúmulo desses elementos tóxicos nos solos decorre da deposição inadequada de efluentes domésticos, resíduos sólidos e/ou uso de agrotóxicos inapropriados para o manejo na agricultura. Uma alternativa para redução desses efeitos e melhor compreensão do comportamento desses metais no solo é o estudo de adsorção. Este trabalho objetivou investigar a capacidade de adsorção simultânea de $\mathrm{Cu} 2+$ e $\mathrm{Cd} 2+$ em ambientes naturais e antropizados localizados na Baixada Maranhense e verifica qual isoterma de adsorção foi mais adequada para esse estudo. Foram determinadas as características físico-químicas e as concentrações dos metais potencialmente tóxicos por espectrometria de absorção atômica por chama (FAAS), além disso, todos os dados foram plotados para os modelos de Langmuir e Freundlich. Os resultados indicaram que a capacidade máxima de adsorção foi favorável ao Cu2+e ambas as isotermas se adequaram ao estudo de adsorção, contudo, constatou-se que o modelo de Langmuir obteve melhor proximidade com os dados experimentais. Os parâmetros de Csm e b apresentaram que em ambiente natural, o metal Cu2+ foi retido nos solos em medidas com diferentes valores de energias de ligação, explicadas pela afinidade da superfície do adsorvente com o adsorvato.

\section{Simultaneous adsorption and competitiveness of cadmium and copper in natural and man-made soils in the city of Cajapió, Baixada Maranhense}

\begin{abstract}
Soil contamination by potentially toxic metals has caused damage to the environment and human health in recent decades. The accumulation of these toxic elements in the soil results from the inadequate deposition of domestic effluents, solid waste and/or the use of inappropriate pesticides for management in agriculture. An alternative to reduce these effects and better understand the behavior of these metals in the soil is the study of adsorption. This work aimed to investigate the capacity of simultaneous adsorption of $\mathrm{Cu} 2+$ and $\mathrm{Cd} 2+$ in natural and anthropized environments located in the Baixada Maranhense and to verify which adsorption isotherm was more suitable for this study. The physico-chemical characteristics and concentrations of potentially toxic metals were determined by flame atomic absorption spectrometry (FAAS), in addition, all data were plotted for the Langmuir and Freundlich models. The results indicated that the maximum adsorption capacity was favorable to $\mathrm{Cu} 2+$ and both isotherms were adapted to the adsorption study, however, it was found that the Langmuir model obtained better proximity to the experimental data. The parameters of Csm and b showed that in a natural environment, the metal Cu2 + was retained in the soils in measurements with different values of binding energies, explained by the affinity of the surface of the adsorbent with the adsorbate.
\end{abstract}

Keywords: Potentially toxic metals; Isotherms; Adsorption.

Topic: Desenvolvimento, Sustentabilidade e Meio Ambiente

Reviewed anonymously in the process of blind peer.

Jonas Juliermerson Silva Otaviano id

Universidade Estadual da Região Tocantina do Maranhão, Brasil http://lattes.cnpq.br/0354528480258226

http://orcid.org/0000-0002-5605-6690

juliermersonsantos@gmail.com

Jéssica Mesquita do Nascimento (iD

Universidade Federal do Rio de Janeiro, Brasil

http://lattes.cnpq.br/6385346174406072

http://orcid.org/0000-0002-3547-6653

jessicanascimento@eq.ufrj.br

José Roberto Pereira de Sousa (ii)

Universidade Estadual do Maranhão, Brasil

http://lattes.cnpq.br/4715196905783353

http://orcid.org/0000-0002-9212-1103

irszoo@hotmail.com

DOI: 10.6008/CBPC2179-6858.2020.005.0046
Received: 10/08/2020

Approved: 21/09/2020

Jorge Diniz de Oliveira (iD)

Universidade Estadual da Região Tocantina do Maranhão, Brasil

http://lattes.cnpq.br/5362473302100013

http://orcid.org/0000-0001-9421-0524

jzinid@hotmail.com
Referencing this:

OTAVIANO, J. J. S.; NASCIMENTO, J. M.; SOUSA, J. R. P.; OLIVEIRA, J. D. Adsorção simultânea e competitividade de cádmio e cobre em solos naturais e antropizados da cidade de Cajapió, Baixada Maranhense. Revista Ibero Americana de Ciências Ambientais, v.11, n.5, p.510518, 2020. DOI: http://doi.org/10.6008/CBPC21796858.2020 .005 .0046 


\section{INTRODUÇÃO}

A expansão de atividades agrícolas com aplicação inadequada de agrotóxicos, aumento no nível de consumo da população e disposição de resíduos sólidos, teve como consequência impactos negativos na natureza em escala global. No Brasil, são gastos 1 bilhão de dólares ao ano em recuperação de áreas contaminadas, sendo a região sudeste, precisamente nos estados de São Paulo, Minas Gerais e Rio de Janeiro, com 5351, 578 e 271 áreas identificadas, respectivamente (MAXIMINIANO et al., 2016).

Há necessidade de conhecer o comportamento adsortivo de metais nos solos, essa reação complexa é responsável pela mobilidade e toxicidade dos mesmos. A compreensão sobre a retenção de um metal potencialmente tóxico em solos pode produzir informações sobre biodisponibilidade e auxiliar em estratégias de intervenção que minimizem impactos nocivos ao ambiente (SENA, 2018). O estudo do processo de adsorção é de indispensável para o entendimento da transferência dos metais da fase líquida para a sólida, sendo que a mesma, controla a solubilidade e biodisponibilidade através de suas características (USMAN, 2008).

As isotermas de Langmuir e Freundlich têm sido as mais utilizadas para estudos de adsorção de metais potencialmente tóxicos em solos brasileiros (COTTA et al., 2020; MATTOS et al., 2016; GONÇALVES et al., 2013; MOREIRA et al., 2005). O processo de adsorção é uma ação de deslocamento de massa que analisa a habilidade de determinados sólidos a capacidade de concentrar na superfície determinadas substâncias existentes em fluidos líquidos ou gasosos que facilitem a separação dos componentes desses fluidos (NASCIMENTO et al., 2014).

Uma das formas de estudar o fenômeno de adsorção de metais nos solos tem sido o uso de isotermas de adsorção, segundo Gonçalves et al. (2013), os modelos propostos por Langmuir e Freundlich são mais utilizados para descrever o comportamento dos metais no solo. O objetivo do presente trabalho é verificar a capacidade de adsorção simultânea de $\mathrm{Cu}^{2+} \mathrm{e} \mathrm{Cd}^{2+}$ em solos da Baixada Maranhense e analisar se entre as isotermas utilizadas (Langmuir e Freundlich) qual o modelo que melhor descreve a adsorção dos metais no solo, nas condições estudadas.

\section{MATERIAIS E MÉTODOS}

\section{Aquisição das amostras e análise de solo}

Neste trabalho, as amostras foram coletadas de três diferentes zonas fitogeográficas do estado do Maranhão. Cada área representa uma unidade amostral, que apresentam uma distância mínima de $2 \mathrm{~km}$ uma da outra. A escolha das áreas antropizadas foi baseada na presença de impactos antrópicos decorrentes de desmatamento para plantação de pastagem e pela ocorrência de gados e bubalinos. A figura a seguir mostra a localização da cidade de Cajapió e suas coordenadas geográficas nos pontos de coleta das amostras entre os ambientes naturais e antropizados. 


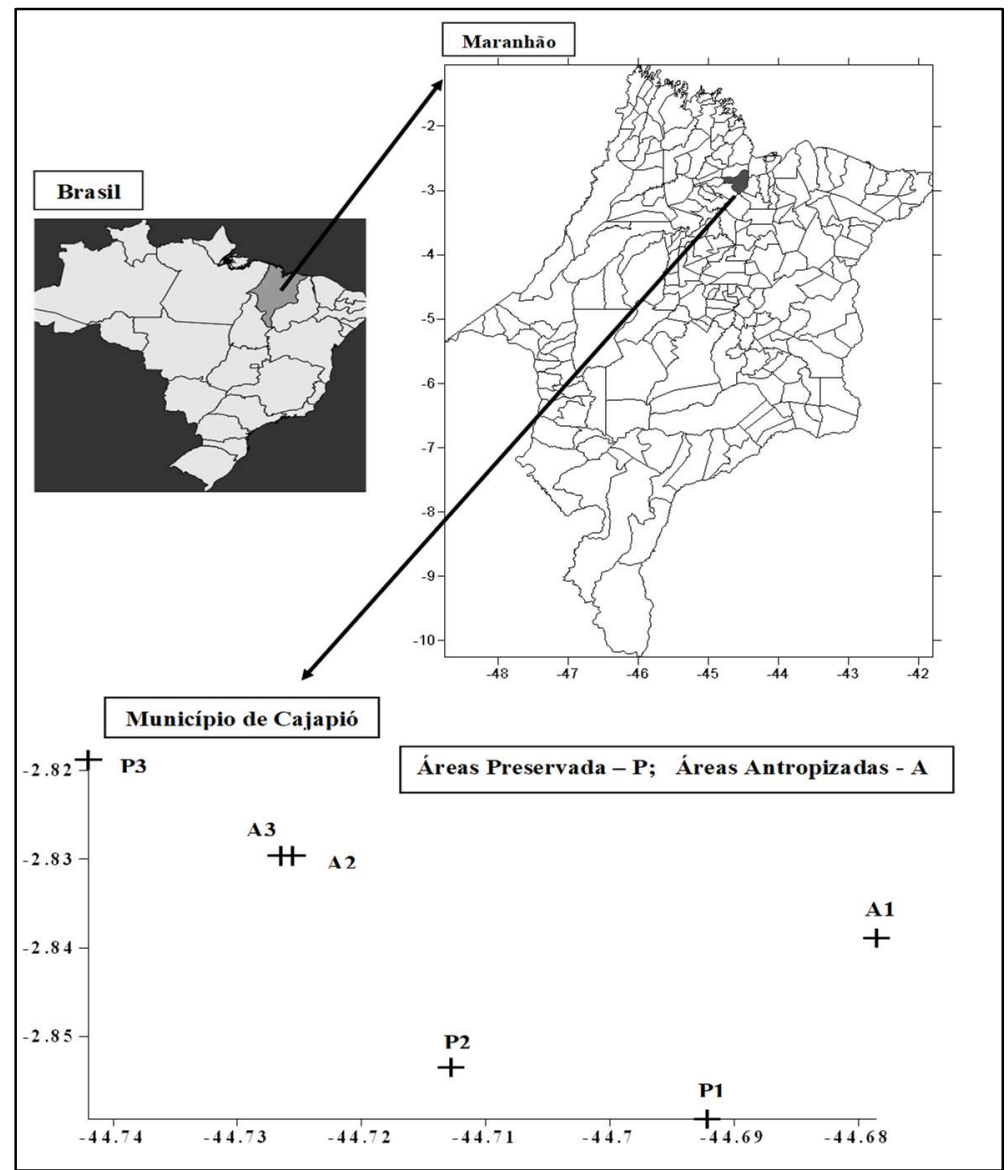

Figura 1: Localização do município e pontos amostrais (Arcgis 6.1; Qgis 3.12.0).

As amostras foram transferidas para bandeja plástica e foram secas em temperatura ambiente. Decorrido o tempo de secagem, as amostras foram descompactadas e trituradas em gral de porcelana, e posteriormente, fracionadas utilizando-se peneiras de $0,35 \mathrm{~mm}$. As análises de $\mathrm{pH}$, determinação de material orgânico e carbono orgânico foram realizadas com base nos métodos de análise de solos da Embrapa (2011).

\section{Método para os ensaios de adsorção}

Foram preparadas soluções-padrão (SpecSol) de concentrações crescentes de $\mathrm{CdCl}_{2}$ e $\mathrm{CuCl}_{2}$ e uma solução-padrão de $\mathrm{CaCl}_{2}$ a $0,01 \mathrm{~mol} \mathrm{~L}^{-1}$. As soluções-padrão dos metais empregados foram preparadas na proporção solo: solução de 1:30, considerando as concentrações iniciais dos respectivos metais na solução de equilíbrio iguais a 2,5, 5, 10, 14, 22, 30, $40 \mathrm{mg} \mathrm{L}^{-1}$. Foram pesadas alíquotas de 1,000 g de solo com granulometria de $0,35 \mathrm{~mm}$ em erlenmeyer, em seguida foi adicionado $20 \mathrm{~mL}$ de solução de $\mathrm{CaCl}_{2}$ 0,01 mol L-

1. O experimento foi realizado em triplicata em $\mathrm{pH}$ natural do solo.

Todas as amostras foram submetidas agitação constante de 200 rpm por 24 horas, após isso, adicionado às amostras $10 \mathrm{~mL}$ das soluções-padrão do metal e filtrados em membrana de acetato filtrante 0,45 mm, condicionadas em frasco de plástico escuro, fora guardado sob refrigeração até o momento da determinação das espécies metálicas. A quantificação no metal na solução filtrada foi feita utilizando-se o Espectrômetro de Absorção Atômica por Atomização por Chama (FAAS), no comprimento de onda do metal específico. 
A quantidade de cobre adsorvida no solo foi calculada de acordo com a diferença de concentração na solução anteriormente e posteriormente ao experimento de adsorção, de acordo com o balanço de massa representado na equação (1)

$$
\begin{aligned}
& \mathbf{q e}=[(\mathbf{C i}-\mathbf{C f}) * \mathbf{V s}] / \mathbf{m s} \mathbf{( 1 )} \\
& \text { qe é concentração de metal sorvido }\left(\mathrm{mg} \mathrm{g}^{-1}\right) ; \\
& \text { Ci é a concentração inicial da solução de equilíbrio }\left(\mathrm{mg} \mathrm{L}^{-1}\right) ; \\
& \text { Cf é a concentração final da solução de equilíbrio }\left(\mathrm{mg} \mathrm{L}^{-1}\right) ;
\end{aligned}
$$

Vs é o volume da solução de equilíbrio (L); ms é a massa de solo da amostra (g).

Os resultados experimentais foram ajustados aos modelos de Langmuir (Equação 2) e de Freundlich (Equação 3).

$$
\begin{aligned}
& q_{e}=\frac{q_{o} K_{L} C_{e}}{1+K_{L} C_{e}} \\
& q_{e}=K_{f} C_{e}^{1 / n}
\end{aligned}
$$

q0 - capacidade máxima de adsorção $\left(\mathrm{mg} \mathrm{g}^{-1}\right)$;

$\mathrm{K}_{\mathrm{L}}$ - constante relacionada à energia de ligação do metal no solo (mg $\left.\mathrm{L}^{-1} 1\right)$;

$\mathrm{K}_{\mathrm{f}}$ - constante de Freundlich (mg g-1);

$\mathrm{n}$ - parâmetro de afinidade do solo pelo soluto (adimensional).

Com a finalidade de delinear a curva de adsorção foi traçado a concentração final na solução de equilíbrio (eixo $\mathrm{x}$ ) versus a quantidade de soluto adsorvido no solo (eixo y). Com base nas linearizações das isotermas, foi plotado para a isoterma de Langmuir a concentração final do soluto na solução de equilíbrio (eixo $\mathrm{x}$ ) versus a concentração final do soluto na solução de equilíbrio sobre a quantidade de soluto adsorvido no solo. Quando uma linha reta é ajustada a estes dados, o modelo de Langmuir foi considerado adequado (CAMPBELL et al., 1995).

A capacidade máxima de adsorção e a afinidade do adsorvente pela superfície foram calculados conforme equação matemática linearizada e coeficientes de regressão linear e angular. Para linearização de Freundlich foi plotado o logaritmo da concentração final do soluto na solução de equilíbrio (eixo x) versus o logaritmo da quantidade de soluto adsorvido no solo, foram tomados os coeficientes de regressão linear e angular e a equação linearizada para calcular suas constantes. Os dados obtidos para a capacidade de adsorção foram submetidos à análise estatística de variância em função das concentrações das soluções.

\section{Análises estatísticas}

Todos os resultados analíticos foram obtidos em triplicata, sendo as médias comparadas por teste Tukey a 5\% de probabilidade. As análises estatísticas foram realizadas no IBM SPSS Statistics (ver. 22.0, IBM Corp., Armonk, Nova York, EUA).

\section{RESULTADOS E DISCUSSÃO}

\section{Características físico-químicas do solo}

Os dados das Tabelas 1 e 2 apresentam os atributos físico-químicos dos solos analisados. Ao comparar as duas áreas verificou-se que as ações antrópicas influenciaram os teores da matéria orgânica nos solos estudados, os valores da matéria orgânica e carbono orgânico mostram-se superiores na área natural. Estudos de alguns autores mostram que o carbono orgânico é uma das variáveis que apresenta maior

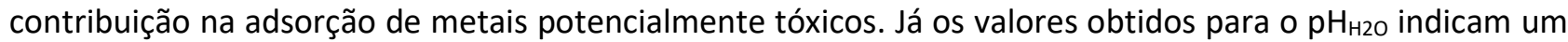


solo ácido (3,5 a 5,0) (GASTALHO et al., 2009). Os valores do $\mathrm{pH}$ em $\mathrm{KCl}\left(1 \mathrm{~mol} \mathrm{~L}^{-1}\right)$ foram inferiores aos observados para o pH em água indicando que o $\mathrm{Cl}^{-}$deslocou menos quantidades de $\mathrm{OH}^{-}$em relação a $\mathrm{H}^{+}$ deslocado pelo $\mathrm{K}^{+}$(SHINZATO et al., 2015).

Os valores $\Delta \mathrm{pH}\left(\Delta \mathrm{pH}=\mathrm{pH}_{\mathrm{KCl}}-\mathrm{pH}_{\mathrm{H} 2 \mathrm{O}}\right)$ são utilizados para se obter uma estimativa da carga líquida do solo. Os dados obtidos para o $\Delta \mathrm{pH}$ nas Tabelas 1 e 2 mostram que em ambas as áreas há predominância de cargas líquidas negativas no solo, portanto, no solo de Cajapió a capacidade de troca catiônica (CTC) supera a capacidade de troca iônica (CTA) em condições de pH natural (FERNANDES et al., 2008). Logo, entende-se que o pH tem importância como fator chave em controlar a adsorção de metais, sendo que solos de pH mais elevado tendem a um aumento da capacidade adsortiva dos mesmos.

Os solos analisados apresentaram características semelhantes a identificação e classificação da Embrapa Solos (2006), sendo geralmente solo ácido, mal drenado, com predomínio de cores pálidas em complexos de cores amarelas e avermelhadas.

Tabela 1: Propriedades físico-químicas do solo em ambiente natural.

\begin{tabular}{l|l}
\hline Atributos & Valores médios \\
\hline M.O \% & 13,14 \\
C.O \% & 7,61 \\
p.H $\mathrm{H}_{\mathrm{HO}}$ & $4,13 \pm 0,094$ \\
p.H & $2,93 \pm 0,051$ \\
$\Delta \mathrm{pH}$ & $-1,2$ \\
\hline
\end{tabular}

M.O\%= Matéria Orgânica; $\mathrm{C} . \mathrm{O} \%=$ Carbono orgânico $\mathrm{pH} \mathrm{H}_{20}=$ média \pm desvio padrão de $\mathrm{pH}$ em água; $\mathrm{pH} \mathrm{kcl}=$ média \pm desvio padrão de $\mathrm{pH}$ em cloreto de potássio; $\Delta \mathrm{pH}$ :Variação do $\mathrm{pH}$.

Tabela 2: Propriedades físico-químicas do solo em ambiente antropizado.

\begin{tabular}{l|l}
\hline Atributos & Valores médios \\
\hline M.O \% & 8,78 \\
C.O \% & 5,09 \\
p. $\mathrm{H}_{\mathrm{H} 2 \mathrm{O}}$ & $4,01 \pm 0,078$ \\
p. $\mathrm{H}_{\mathrm{KCl}}$ & $2,92 \pm 0,12$ \\
$\Delta \mathrm{pH}$ & $-1,09$ \\
\hline
\end{tabular}

M.O\%= Matéria Orgânica; C.O\%= Carbono orgânico $\mathrm{pH}_{\mathrm{H} 2 \mathrm{O}}=$ média \pm desvio padrão de $\mathrm{pH}$ em água; $\mathrm{pH} \mathrm{Kcl}=\mathrm{média} \pm$ desvio padrão de $\mathrm{pH}$ em cloreto de potássio; $\Delta \mathrm{pH}$ :Variação do $\mathrm{pH}$.

\section{Isotermas de adsorção e competitividade}

As isotermas de Langmuir e Freundlich são usadas, por meio de equações, para descrever a capacidade máxima de adsorção e o comportamento dos dados obtidos através de experimentos (NASCIMENTO et al., 2014). O ajuste dos resultados experimentais a modelos de adsorção fornece informações importantes sobre a capacidade de retenção e a força pela qual o adsorvido está aderido ao solo.

Observando a Figura 2 verifica-se que à medida que a concentração inicial aumentou, maiores quantidades de $\mathrm{Cd}^{2+}$ e $\mathrm{Cu}^{2+}$ foram adsorvidas indicando não haver saturação dos sítios de adsorção. De modo geral, em ambas as áreas investigadas, o $\mathrm{Cu}^{2+}$ ficou ligeiramente retido no solo acima do $\mathrm{Cd}^{2+}$. Isto pode ser explicado pela energia de ligação que estes elementos apresentam. $\mathrm{Na}$ retenção do $\mathrm{Cu}^{2+}$, ocorrem predominância de força eletrostáticas, enquanto que o $\mathrm{Cd}^{2+}$ pode ser adsorvido por atração eletrostática na dupla camada difusa (adsorção não específica) ou pela complexação de adsorção específica, meio em que o 
elemento se liga à matriz do solo através de ligações covalentes, o que reduz sua mobilidade (SELIM, 1992). A relação entre os teores de cobre e cádmio na solução de equilíbrio e os adsorvidos mostra que os solos em ambiente natural e antropizado apresentam perfis semelhantes para suas isotermas, classificadas como do tipo C, o que indica alta afinidade de adsorção. A capacidade de adsorção dos metais pode variar conforme o tipo de material adsorvido, convém lembrar que a superfície de um solido é composta por um número considerável de sítios. A figura 2 objetiva verificar a competitividade de Cobre e Cádmio mediante aos modelos de Freundlich e Langmuir.
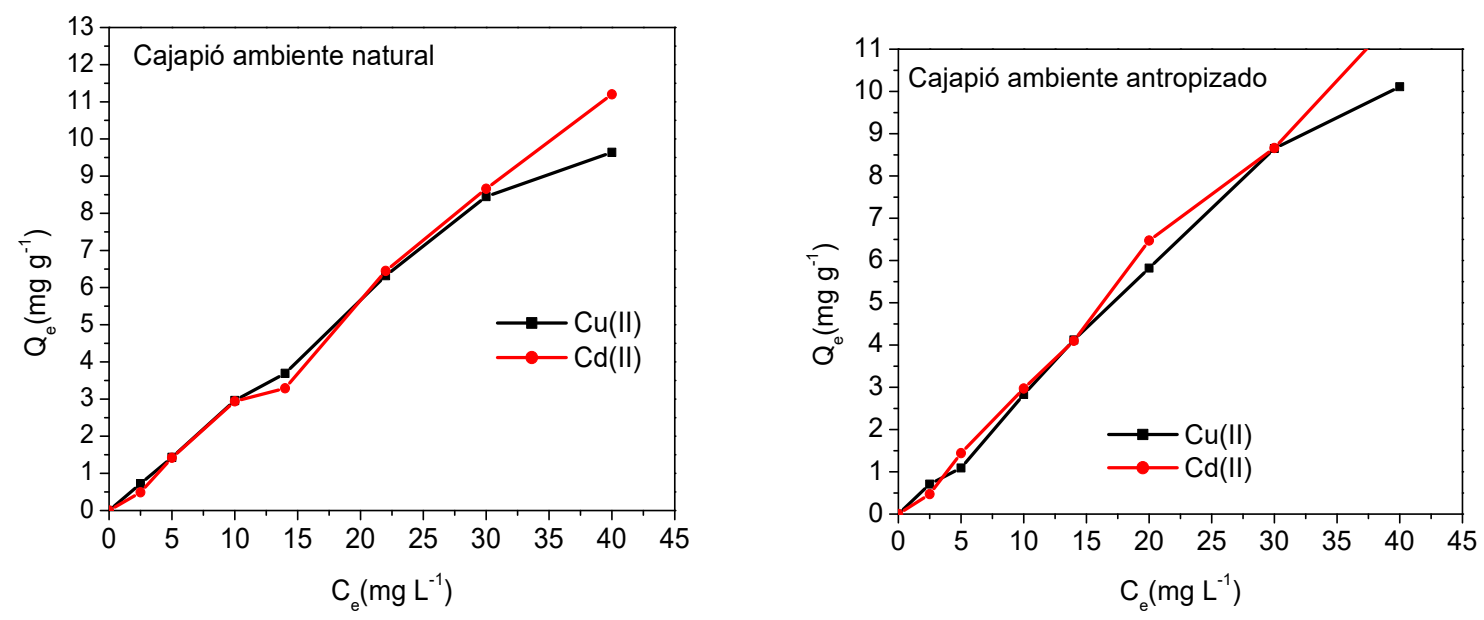

Figura 2: Isoterma de adsorção de Cu (II) e Cd (II) no sistema completivo para o solo de Cajapió em ambiente natural e antropizado.

Para estudar a propriedade de sorção de um metal pelos constituintes do solo é empregado o conceito de coeficiente de distribuição $\left(K_{d}\right)$. O coeficiente de distribuição é definido como a relação entre a concentração do metal na fase sólida e a concentração do metal na solução de equilíbrio: $K d=\frac{Q e}{C e}$ ( $\mathrm{D}^{\prime}$ AGOSTINHO et al., 2006). O K de um metal no solo está ligado as características físico-químicas do solo (pH, textura, óxidos e matéria orgânica.) e da solução do solo (carbono orgânico dissolvido e íons) e do metal (concentração e forma química). Nesse modelo é importante considerar os valores de $\left(\mathrm{K}_{\mathrm{F}}\right)$ coeficientes de Freundlich, coeficientes de distribuição $\left(K_{d}\right)$, além dos valores de $(R)$ regressão linear.

Considerando a comparação entre os modelos de isotermas de adsorção, observou-se que o modelo de Langmuir obteve melhor ajuste, pois os parâmetros mostrados nas isotermas de Freundlich (tabela 4) mostraram que valores de $\mathrm{N}_{\mathrm{F}}$ são menores que 1 , contudo, os valores de $\mathrm{K}_{d}$ indicaram afinidade dos sítios de adsorção do solo pelo $\mathrm{Cu}^{2+}$, assim, é possível verificar que ambos os modelos se adequaram ao estudo de adsorção, sendo o modelo de Langmuir com melhor ajuste experimental. Resultados semelhantes com relação a capacidade máxima de adsorção foram verificados em estudos de Barros (1994, citado por LINHARES et al., 2010), utilizando-se as isotermas de Langmuir e Freundlich na adsorção de cobre, mesmo em solos com características diferentes, o cobre tem alto potencial de interação química com componentes minerais e orgânicos no solo, e por isso, apresenta tendência no acúmulo do $\mathrm{Cu}^{2+}$ em sua superfície.

De acordo com Linhares et al. (2008) o modelo de Freundlich proporcionou baixa capacidade máxima de adsorção do $\mathrm{Cd}^{2+}$ e maior afinidade do cádmio para as classes de solo Chernossolo Argilúvico Carbonático 
saprolítico e Cambissolo Háplico Tb eutrófico latossólico. Em comparação a este trabalho, que apresenta um solo com características diferentes, o cádmio obteve $K_{d}<0$, ou seja, as concentrações do elemento adquiridas na solução de equilíbrio mostram menor afinidade do cádmio e menor coeficiente de distribuição em comparação ao $\mathrm{Cu}^{2+}$ nos solos estudados em ambiente natural e antropizado.

Os valores das constantes foram calculados e estão representados na Tabela 3 e 4, respectivamente. A capacidade máxima de adsorção (Csm) para o cobre nas isotermas para Langmuir na Tabela 3, mostram que o maior valor ocorreu na área natural $\left(10,64 \mathrm{mg} \mathrm{g}^{-1}\right)$, com energia de ligação sendo 1,05 mg L-1; O grau de desenvolvimento no processo de adsorção foi de 0,023. O coeficiente de partição para o processo de sorção em Freundlich a 0,28 (Tab. 4) e a reatividade dos sítios de ligação do solo com valor de 0,45. Na área antropizada para o modelo Langmuir (Tabela 3) verificou-se a energia de ligação de $0,74 \mathrm{mg} \mathrm{L}^{-1}$, o que indica menor capacidade máxima de adsorção em solo antropizado, o $R_{\mathrm{L}}$ (Fator de separação) foi de 0,032. 0 coeficiente de partição para o processo de sorção em Freundlich a 0,40 e a reatividade dos sítios de ligação do solo com 0,061. Para Nascimento et al. (2014), os fenômenos relacionados a adsorção são resultantes da combinação entre forças envolvidas na adsorção física e química, relacionados as propriedades dos adsorventes, adsorvatos, $\mathrm{pH}$ do meio e natureza do solvente.

Os valores das isotermas de Langmuir mostraram que o alto nível da capacidade máxima de adsorção está relacionado ao teor de carbono orgânico e matéria orgânica, fatores que contribuíram para o estudo de adsorção neste trabalho, na composição de material húmico foi um dos principais fatores que interferiram no processo de adsorção. Os valores de Csm e b indicaram que em ambiente natural, o $\mathrm{Cu}^{+2}$ foi retido nos solos em proporções com diferentes valores de energias de ligações, que são justificadas pela afinidade da superfície do adsorvente com o adsorvato. Dos solos investigados, aqueles que tiveram maiores níveis de adsorção foram de área natural, indicando assim, que esses solos são os mais adequados para disposição dessa espécie metálica. $\mathrm{O}$ valor de $\mathrm{R}_{\mathrm{L}}$ maior que zero e menor que um indicam que adsorção é favorável e espontânea. De acordo com os valores supracitados, o $\mathrm{R}_{\mathrm{L}}$ obtido para o $\mathrm{Cu}^{2+}$ em todos os ambientes investigados são favoráveis e espontâneos na faixa de concentração estudada para o modelo de Langmuir. No entanto, os valores de $N_{f}$ para o modelo de Freundlich foram menores que $1\left(N_{f}<1\right)$.

Os valores dos parâmetros de $\mathrm{Cd}^{2+}$ em ambiente natural para as isotermas de Langmuir (Tab.3) apresentaram o Csm de 2,57 $\mathrm{mg} \mathrm{g}^{-1}$; b em 6,64 $\mathrm{mg} \mathrm{L}^{-1}$; $\mathrm{R}_{\mathrm{L}}$ com valor de 0,0037. Em ambiente antropizado, verificou-se os seguintes resultados: Csm de 1,97 $\mathrm{mg} \mathrm{g}^{-1}$; $\mathrm{b}$ em 3,84 $\mathrm{mg} \mathrm{L}^{-1} ; \mathrm{R}_{\mathrm{L}}$ com valor de 0,0065 . Com relação as isotermas de Freundlich (Tab.4), os resultados dos parâmetros de $\mathrm{Cd}^{2+}$ em área natural foram de $\mathrm{K}_{\mathrm{F}}$ a $0,42 \mathrm{mg} \mathrm{L}^{-1} ; \mathrm{Nf}$ em 0,393 $\mathrm{g} \mathrm{L}^{-1} ; \mathrm{K}_{\mathrm{d}}$ a 0,42. Em ambiente antropizado, os valores foram em $\mathrm{K}_{\mathrm{f}}$ igual a 0,37 $\mathrm{mg} \mathrm{L}^{-1} ; \mathrm{N}_{\mathrm{F}}$ com valor de 0,$115 ; \mathrm{K}_{\mathrm{d}}$ a 1,10 . Referente aos resultados de Langmuir, o grau de desenvolvimento do processo de adsorção $\left(R_{L}\right)$ descreve favoravelmente a adsorção, uma vez que o valor encontrado foi maior que 0 e menor que 1 . Tendo como base esses resultados, entende-se, portanto, que a capacidade de adsorção experimental segue no sentido $\mathrm{Cu}^{2+}>\mathrm{Cd}^{2+}$, indica que, esse resultado está associado a estabilidade na solução que o íon $\mathrm{Cd}^{2+}$ apresenta, logo, esse fenômeno inibe o favorecimento do equilíbrio de adsorção o $\mathrm{Cu}^{2+}$ por sua vez mostra maior constante de quebra de $\mathrm{H}_{2} \mathrm{O}$, o que influencia na instabilidade da quebra de 
solução (NASCIMENTO et al., 2014). Os maiores valores de $R_{\mathrm{L}}$ para o cobre indicam que os solos estudados apresentam afinidade pelo cobre.

Tabela 3: Parâmetros obtidos na isoterma adsorção de Langmuir.

\begin{tabular}{l|l|l|l|l|l|l|l|l}
\hline Áreas & $\mathrm{R}$ & $\mathrm{Csm}\left(\mathrm{mg} \cdot \mathrm{g}^{-1}\right)$ & $\mathrm{b}\left(\mathrm{mg} \mathrm{L}^{-1}\right)$ & \multicolumn{2}{l}{$\mathrm{R}_{\mathrm{L}}$} \\
\hline & $\mathrm{Cu}^{2+}$ & $\mathrm{Cd}^{2+}$ & $\mathrm{Cu}^{2+}$ & $\mathrm{Cd}^{2+}$ & $\mathrm{Cu}^{2+}$ & $\mathrm{Cd}^{2+}$ & $\mathrm{Cu}^{2+}$ & $\mathrm{Cd}^{2+}$ \\
\hline Natural & 0,85 & 0,18 & 10,64 & 2,57 & 1,05 & 6,64 & 0,023 & 0,004 \\
\hline Antropizada & 0,76 & $-0,05$ & 8,62 & 1,97 & 0,74 & 3,85 & 0,032 & 0,007 \\
\hline
\end{tabular}

$\mathrm{R}=$ regressão linear, Csm= Capacidade máxima de adsorção em (mg.g $\left.\mathrm{g}^{-1}\right), \mathrm{b}=$ Coeficiente Angular em $\left(\mathrm{mg} \mathrm{L}^{-1}\right), \mathrm{R}_{\mathrm{L}}=\mathrm{grau}$ de desenvolvimento do processo de adsorção.

Tabela 4: Parâmetros obtidos na isoterma adsorção de Freundlich.

\begin{tabular}{l|l|l|l|l|l|l|l|l}
\hline & $\mathrm{R}$ & \multicolumn{2}{l}{$\mathrm{K}_{\mathrm{f}}\left(\mathrm{mg} \mathrm{L}^{-1}\right)$} & $\mathrm{N}_{\mathrm{f}}$ & $\mathrm{K}_{\mathrm{d}}$ \\
\hline Áreas & $\mathrm{Cu}^{2+}$ & $\mathrm{Cd}^{2+}$ & $\mathrm{Cu}^{2+}$ & $\mathrm{Cd}^{2+}$ & $\mathrm{Cu}^{2+}$ & $\mathrm{Cd}^{2+}$ & $\mathrm{Cu}^{2+}$ & $\mathrm{Cd}^{2+}$ \\
\hline Natural & 0,75 & 0,72 & 0,28 & 0,42 & 0,46 & 0,39 & 1,15 & 0,42 \\
\hline Antropizada & 0,07 & 0,08 & 0,41 & 0,37 & $-0,06$ & $-0,12$ & 1,53 & 0,10 \\
\hline $\mathrm{R}=$ regressão linear, \\
$\mathrm{NF}=$ indica a reatividade dos sítios de ligação do solo em $\left(\mathrm{g} \mathrm{L}_{\mathrm{f}}^{-1}\right), \mathrm{Kd}_{\mathrm{d}}=\mathrm{O}$ Coeficiente de Distribuição $\left(\mathrm{L} \mathrm{kg}^{-1}\right)$
\end{tabular}

Mesmo com baixa concentração nas dosagens, os elementos estudados apresentam afinidade entre os constituintes do solo. No processo competitivo, a composição iônica da solução exerce amplo efeito na adsorção de íons nas partículas dos solos. No entanto, o que causa esse efeito é a competitividade entre os metais. De acordo com a tabela 5, não ocorreu diferenças significativas entre as áreas naturais e antropizadas em baixas concentrações, no entanto, houve diferença entre os metais. Entende-se que a comparação estatística indica que os solos tiveram capacidade de adsorção referente aos metais estudados, isto é, os solos tanto de área natural quanto antropizada tem capacidade de concentrar $\mathrm{Cu}^{2+}$ e $\mathrm{Cd}^{2+}$ em sua superfície, demonstrando afinidade entre a superfície dos adsorventes com os adsorvatos. Nesse estudo, foi verificado que o Cu descreve favoravelmente na adsorção se comparado ao $\mathrm{Cd}^{2+}$, de outro modo, significa afirmar que o metal foi substituído pelo $\mathrm{Cu}^{2+}$ no sistema competitivo.

Tabela 5: Comparação estatística da capacidade de adsorção de $\mathrm{Cu}^{2+}$ e $\mathrm{Cd}^{2+}$ do solo de Cajapió em área natural e antropizada nas diferentes dosagens.

\begin{tabular}{|c|c|c|c|c|c|c|c|c|}
\hline \multirow{2}{*}{ Área } & \multicolumn{8}{|c|}{ Dosagem adicionada $\left(\mathrm{mg} \mathrm{L}^{-1}\right)$} \\
\hline & & 2,5 & 5 & 10 & 14 & 22 & 30 & 40 \\
\hline \multirow{2}{*}{ Natural } & $\mathrm{Cu}$ & $0,72 \mathrm{aE}$ & $1,43 \mathrm{aE}$ & $2,95 \mathrm{aD}$ & $3,69 a b D$ & $6,32 \mathrm{aC}$ & $8,45 \mathrm{aB}$ & $10.11 \mathrm{bA}$ \\
\hline & $\mathrm{Cd}$ & $0,49 \mathrm{aF}$ & $1,42 \mathrm{aE}$ & $2,94 \mathrm{aD}$ & $3,29 \mathrm{bD}$ & $6,45 \mathrm{aC}$ & $7,66 \mathrm{bB}$ & $11,20 \mathrm{aA}$ \\
\hline \multirow{2}{*}{ Antropizada } & $\mathrm{Cu}$ & $0,72 \mathrm{aF}$ & $1,09 \mathrm{aF}$ & $2,83 \mathrm{aE}$ & $4,12 \mathrm{aD}$ & $6,15 \mathrm{aC}$ & $8,65 \mathrm{aB}$ & $10,10 \mathrm{bA}$ \\
\hline & $\mathrm{Cd}$ & $0,47 \mathrm{aF}$ & $1,44 \mathrm{aE}$ & $2,97 \mathrm{aCD}$ & $3,20 \mathrm{bC}$ & $5,29 \mathrm{bB}$ & $2,12 \mathrm{cDE}$ & $9,35 \mathrm{cA}$ \\
\hline
\end{tabular}

As médias seguidas pela mesma letra minúscula na coluna e maiúscula na linha não diferem estatisticamente entre si. Foi aplicado o Teste de Tukey ao nível de $5 \%$ de probabilidade.

A verificação da capacidade de adsorção dos metais estudados possibilitou compreensão sobre o comportamento e competitividade desses elementos no processo de adsorção simultânea. Todavia, alguns parâmetros físico-químicos não realizados neste trabalho, como a CTC, porosidade, PZC e análises de grupos funcionais presentes no adsorvente são necessários para melhor entendimento do sistema competitivo.

\section{CONCLUSÕES}

Comparado ao $\mathrm{Cd}^{2+}$, o $\mathrm{Cu}^{2+}$ descreve favoravelmente na adsorção, de outro modo, significa que o $\mathrm{Cd}^{2+}$ pode ser substituído pelo $\mathrm{Cu}$ no sistema competitivo. Os tipos de solos estudados tiveram capacidade 
máxima de adsorção maior para cobre com relação ao cádmio, soma-se a isso, que os solos investigados interferiram nos resultados, por apresentar condições de um solo mal drenado e com pH ácido. Os coeficientes de determinação $\mathrm{R}$ indicaram que os dados experimentais se ajustaram melhor ao modelo de Langmuir do que ao de Freundlich para o $\mathrm{Cu}^{2+}$. Ambos os modelos se adequaram ao estudo de adsorção, sendo o modelo de Langmuir com melhor ajuste experimental.

\section{REFERÊNCIAS}

COTTA, J. A. P. O.; RODRIGUES, M. C.. Estudo da sorção de chumbo em solos intemperizados e em suas diferentes frações. Research, Society and Development, Itabira v.9, n.4, 2020. DOI: http://dx.doi.org/10.33448/rsd-v9i4.2306

CAMPBELL, L. S.; DAVIES, B. E.. Soil sorption of caesium modelled by the Langmuir and Freundlich isotherms equations. Applied Geochemistry. v.10, p.715-723, 1995. DOI: https://doi.org/10.1016/0883-2927(95)00056-9

D'AGOSTINHO, A.; FLUES, M.. Determinação do coeficiente de distribuição $(\mathrm{Kd})$ de benzo(a)pireno em solo por isotermas de sorção. Química Nova, São Paulo v.29, n.4, 2006. DOI: http://dx.doi.org/10.1590/S010040422006000400006

EMBRAPA. Manual de métodos de análise de solo. Rio de Janeiro: Centro Nacional de Pesquisa de Solos, 2006.

EMBRAPA. Manual de métodos de análise de solo. Rio de Janeiro: Centro Nacional de Pesquisa de Solos, 2011.

FERNANDES, D. J.; GARÓFALES, CHAVES, L. H.; OLIVEIRA, F H. T.; FARIAS, D. R.. Ponto de efeito salino nulo e cargas elétricas de solos do estado da Paraíba. Revista caatinga, Mossoró, v.21, n.2, p.147-155, 2008.

GASTALHO, C.; BARREIROS, I.; RIBEIRO, V.. Determinação do pH e acidez de uma amostra de solo. Monografia (Licenciatura em Ciências Bioanalíticas, Hidrologia e Edafologia) - Universidade de Coimbra, Coimbra, 2009.

GONÇALVES, M. S.; BETTIN, J. P.; SILVA JUNIOR, L. C. S.; SAMPAIO, S. C.; DAL BOSCO, T. C.. Adequação dos modelos de Langmuir e Freundlich na adsorção de cobre em solo argiloso do sul do Brasil. HOLOS, v.4, p.37-44, 2013.

LINHARES, L. A.; FILHO, F. B. E.; BELLIS, V. M.; SANTOS, E. A.; IANHEZ, R.. Utilização dos modelos de Langmuir e Freundlich na adsorção de cobre e zinco em solos brasileiros. Acta Agronômica, v.3, p.303-315, 2010.

LINHARES, L. A.; FILHO, F. B. E.; IANHEZ, R.; SANTOS, E. A.. Aplicação dos modelos de Langmuir e Freundlich na adsorção de cádmio e chumbo em diferentes classes de solos brasileiros. Revista Tecnológica, v.17, p.49-60, 2008.

MAXIMINIANO, A. M. S.; BARBOSA, A. M.; CAVANI, A. C. M.; QUEIROZ, A. P.; GUIMARÃES, C. C.; TEIXEIRA, C. E.; CIRANI, C. B. S.; SACCOCCIO, E. M.; MOTTA, F. G.; SETTI, G.; GARCIA, L, P.; ARAÚJO, M. M.; RISSARDI, M.; BRITO, M.; YOSHIKAWA, N. K.; CUNHA, R. C. A.; MORAES, S. L.; TAVARES, T.; GOMES, T. L.; GUIMARÃES, Y. B. T.. Panorama GAC: Mapeamento da cadeia de gerenciamento de áreas contaminadas. Instituto de Pesquisas Tecnológicas, 2016.

MATTOS, G. A.; AMARAL SOBRINHO, N. M. B.; LIMA, E. S. A.; GUEDES, J. N.; SOUSA, F. F.. Sorção de Cd e Pb nos solos da região do Médio Rio Paraíba - RJ, Brasil. Revista Ciência Agronômica, v.47, n.1, p.1-12, 2016.

MOREIRA, C. S.; ALLEONI, L. R.. Adsorption of Cd, Cu, Ni and $\mathrm{Zn}$ in tropical soils under competitive and non-competitive systems. Scientia Agrícola, v.67, n.3, p.301-307, 2010. DOI: https://doi.org/10.1590/S0103-90162010000300008

NASCIMENTO, R. F.; LIMA, A. C. A.; VIDAL, C. B.; MELO, D. Q.; RAULINO, G. S. C.. Adsorção: aspectos teóricos e aplicações ambientais. Fortaleza: Imprensa Universitária, 2014.

SENA, M. M.. Adsorção individual, competitiva e estudos cinéticos e termodinâmicos de cobre, chumbo e níquel em solos de uma topossequência do cerrado. Tese (Doutorado) - Universidade de Brasília, Brasília, 2018.

SELIM, H. M.. Modelling the transport and retention of inorganics in soils. Advances in Agronomy, v.47, p.331-384, 1992. DOI: https://doi.org/10.1016/S0065-2113(08)60493-9

SHINZATO, M. C.; ANDRADE, V. S.; MARYINS, J. V.; SANTOS, M. M. A.; REBEIRO, V. T.; CARVALHO, F. M. S.. Efeito de lama vermelha nas propriedades eletroquímicas de adsorção de um latossolo vermelho. Revista Instituto Geológico, v.36, n.1, p.51-65, 2015.

USMAN, A. R. A.. The relative adsorption selectivities of $\mathrm{Pb}$, $\mathrm{Cu}, \mathrm{Zn}, \mathrm{Cd}$ and $\mathrm{Ni}$ by soils developed on shale in New Valley, Egypt. Geoderma, v.144, p.334-343, 2008. DOI: http://doi.org/10.1016/j.geoderma.2007.12.004

A CBPC - Companhia Brasileira de Produção Científica (CNPJ: 11.221.422/0001-03) detém os direitos materiais desta publicação. Os direitos referem-se à publicação do trabalho em qualquer parte do mundo, incluindo os direitos às renovações, expansões e disseminações da contribuição, bem como outros direitos subsidiários. Todos os trabalhos publicados eletronicamente poderão posteriormente ser publicados em coletâneas impressas sob coordenação da Sustenere Publishing, da Companhia Brasileira de Produção Científica e seus parceiros autorizados. Os (as) autores (as) preservam os direitos autorais, mas não têm permissão para a publicação da contribuição em outro meio, impresso ou digital, em português ou em tradução. 